\title{
ARTICLE \\ MNK1 inhibitor CGP57380 overcomes mTOR inhibitor-induced activation of eIF4E: the mechanism of synergic killing of human T-ALL cells
}

\author{
Xian-bo Huang ${ }^{1}$, Chun-mei Yang ${ }^{1,2}$, Qing-mei Han ${ }^{1}$, Xiu-jin Ye ${ }^{1}$, Wen Lei ${ }^{1,2,3}$ and Wen-bin Qian ${ }^{1,2,3}$
}

\begin{abstract}
Although the treatment of adult T-cell acute lymphoblastic leukemia (T-ALL) has been significantly improved, the heterogeneous genetic landscape of the disease often causes relapse. Aberrant activation of mammalian target of rapamycin (mTOR) pathway in T-ALL is responsible for treatment failure and relapse, suggesting that mTOR inhibition may represents a new therapeutic strategy. In this study, we investigated whether the mTOR complex 1 (mTORC1) inhibitor everolimus could be used as a therapeutic agent against human T-ALL. We showed that rapamycin and its analog RAD001 (everolimus) exerted only mild inhibition on the viability of Jurkat, CEM and Molt-4 cell lines (for everolimus the maximum inhibition was $<40 \%$ at $100 \mathrm{nM}$ ), but greatly enhanced the phosphorylation of elF4E, a downstream substrate of MAPK-interacting kinase (MNK) that was involved in promoting cell survival. Furthermore, we demonstrated in Jurkat cells that mTOR inhibitor-induced elF4E phosphorylation was independent of insulin-like growth factor-1/insulin-like growth factor-1 receptor axis, but was secondary to mTOR inhibition. Then we examined the antileukemia effects of CGP57380, a MNK1 inhibitor, and we found that CGP57380 (4-16 $\mu \mathrm{M})$ dose-dependently suppressed the expression of both phosphor-MNK1 and phosphor-elF4E, thereby inhibiting downstream targets such as c-Myc and survivin in TALL cells. Importantly, CGP57380 produced a synergistic growth inhibitory effect with everolimus in T-ALL cells, and treatment with this targeted therapy overcame everolimus-induced eIF4E phosphorylation. In conclusion, our results suggest that dual-targeting of mTOR and MNK1/elF4E signaling pathways may represent a novel therapeutic strategy for the treatment of human T-ALL.
\end{abstract}

Keywords: T-ALL; mTOR; MNK1; elF4E; drug-resistance; everolimus; CGP57380

Acta Pharmacologica Sinica (2018) 39:1894-1901; https://doi.org/10.1038/s41401-018-0161-0

\section{INTRODUCTION}

T-cell acute lymphoblastic leukemia (T-ALL) is an aggressive and heterogeneous blood malignancy disease derived from Tcell progenitor cells in the thymus [1]. T-ALL accounts for approximately $10 \%-15 \%$ of pediatric and $25 \%$ of adult ALL cases [2]. With the current intensive chemotherapy regimens, the 5 -year event-free survival (EFS) of children with T-ALL has reached $70 \%-75 \%$, whereas the EFS is $\sim 50 \%$ for adolescents and young adults, and $10 \%$ for those older than 60 years of age [3-5]. Nevertheless, novel therapies aimed at improperly activated signaling pathways, which are involved in cancer cell survival and drug resistance, are still needed to improve the patient outcome in T-ALL.

Among the deregulated signaling pathways that have been identified in T-ALL, the phosphatidylinositol 3-kinase (PI3K)/Akt/ mammalian target of rapamycin (mTOR) pathway has been reported to be active in most patients [6-8]. mTOR is the catalytic subunit of two distinct multi-protein complexes known as mTOR complex 1 (mTORC1) and mTOR complex 2 (mTORC2), both of which have been implicated in T-ALL pathophysiology
$[6,9]$. Recently, it was demonstrated that the inhibition of mTORC1 activity induces developmental blockade in early $\mathrm{T}$ lymphopoiesis and eradicates T-ALL cells [10]. Rapamycin, an allosteric mTORC1 inhibitor, was tested in vitro in pre-clinical models of T-ALL, where it induced apoptosis and/or cell cycle arrest and synergized with chemotherapeutic agents [11-14]. Rapamycin derivatives (rapalogs), such as CCl-779 (temsirolimus) and RAD001 (everolimus), display improved bioavailability compared with rapamycin [15]. On the basis of in vitro studies confirming its antileukemia properties, everolimus has been evaluated recently in a phase II clinical trial in patients with relapsed T-cell lymphoma $(\mathrm{TCL})$. This trial demonstrated a $44 \%$ overall response rate [16]. Additionally, everolimus has entered phase I/II trials for T-ALL, either alone (ClinicalTrials.gov: NCT00081874) or in combination with conventional chemotherapy (NCT00968253; NCT01523977; NCT01403415). However, in a phase $I / I I$ trial of everolimus in combination with HyperCVAD chemotherapy, $29 \%$ of patients with relapsed/refractory $A L L$, including $T-A L L$, had complete remission, indicating that the therapy was moderately effective [17].

${ }^{1}$ Department of Hematology, the First Affiliated Hospital, College of Medicine, Zhejiang University, Hangzhou 310003, China; ${ }^{2}$ Malignant Lymphoma Diagnosis and Therapy

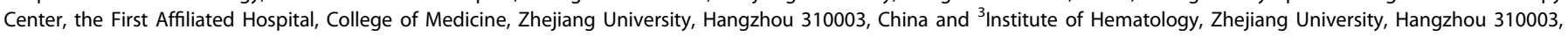
China

Correspondence: Xiu-jin Ye (yxjsunny@163.com) or Wen-bin Qian (qianwb@zju.edu.cn)

These authors are co-first authors: Xian-bo Huang, Chun-mei Yang.

Received: 9 April 2018 Accepted: 30 July 2018

Published online: 8 October 2018 
A family of kinases that are key effectors for MAPK pathways includes the MNK1 and MNK2 kinases, which regulate the phosphorylation of eukaryotic initiation factor 4E (elF4E) [18]. MNK/elF4E plays a significant regulatory role in mRNA translation and malignant transformation. The phosphorylation of elF4E by MNK at Ser209 is implicated in tumorigenesis, such as lymphomagenesis [19]. Lymphomas expressing elF4E are highly resistant to chemotherapy. Importantly, MNK activity does not appear to be essential for normal cell growth [20]. Therefore, targeting this pathway may provide a new therapeutic strategy for lymphoma and leukemia [21-23].

Acquired and inherent drug resistance is a potential concern associated with anticancer small-molecule inhibitors. For example, mTOR inhibitors could hyperactivate Akt because of the existence of feedback loops among mTOR, PI3K, and Akt [24-27], impeding their progression in cancer treatment for use as single agents. Therefore, in this study, we investigated the mTORC1 inhibitor everolimus as a therapeutic agent against T-ALL. We found that everolimus has a limited effect on the proliferation of T-ALL cell lines that is related to the phosphorylation of elF4E, a downstream molecule of MNK that is involved in promoting cell survival [28]. Thus, we next investigated the anti-leukemia effects of everolimus combined with the MNK1 inhibitor CGP57380. Our results demonstrated that eIF4E inhibition might play a functional role in the increased cell death observed in combination therapy and that CGP57380 might antagonize the resistance to mTORC1 inhibitors.

\section{MATERIALS AND METHODS}

Reagents

Rapamycin and everolimus, pan-caspase inhibitor z-VAD-fmk and IGF-1R inhibitor PQ401 were purchased from Selleck Chemicals (Houston, TX, USA). LY294002 was purchased from LKT Laboratories, Inc. (St. Paul, MN, USA). The MNK1 inhibitor 4-amino-5-(4fluoroanilino)-pyrazolo [3, 4-d] pyrimidine (CGP57380) was purchased from Sigma-Aldrich (St. Louis, MO, USA). IGF-1 was purchased from Peprotech (Rocky Hill, NJ, USA). The agents were dissolved in dimethyl sulfoxide (DMSO), and aliquots were stored at $4{ }^{\circ} \mathrm{C}$ or $-20^{\circ} \mathrm{C}$ according to the manufacturer's instructions. Stock solutions of these agents were subsequently diluted with serum-free RPMI-1640 medium immediately before use. In all experiments, the final concentration of DMSO did not exceed $0.1 \%$. Rabbit monoclonal antibodies against mTOR, phosphor-mTORC1 (p-mTORC1) (Ser2448), PI3K-p110a, phosphor-Akt (p-Akt) (Ser473), p70S6K, phosphor-p70S6K (pp70S6K) (Thr389), phosphor-MNK1 (p-MNK1) (Thr197/202), 4EBP1, phosphor-4EBP-1 (p-4EBP1) (Ser65), elF4E, Caspase 3, PARP, $\mathrm{BCl}-2, \mathrm{Mcl}-1$, survivin, $c-M y c, C D K 2$ and $\beta$-actin were purchased from Cell Signaling Technology Inc. (Beverly, MA, USA). Mouse monoclonal antibodies against caspase 8 and caspase 9 were also purchased from Cell Signaling Technology. Rabbit monoclonal antibodies against MNK1 and phosphor-elF4E (p-elF4E) (Ser209) were acquired from Abcam (Cambridge, MA, USA). Rabbit polyclonal antibody against Lamin B1 was purchased from Peprotech.

Cell lines and cell culture

The human T-ALL cell lines Jurkat, CEM and Molt-4 were obtained from the Shanghai Cell Collection (Shanghai, China). Cell lines were cultured in RPMI-1640 (Corning Cellgro, Manassas, VA, USA) supplemented with $10 \%$ heat-inactivated fetal bovine serum (Gibco, Grand Island, NY, USA) at $37^{\circ} \mathrm{C}$ in an incubator with $5 \% \mathrm{CO}_{2}$.

MTT colorimetric survival assay

Cell viability was determined using the 3-(4,5-dimethylthiazol-2yl)-2,5-diphenyl tetrazolium bromide (MTT; Sigma, St. Louis, MO,
USA). Briefly, the cells were seeded in 96-well plates at a density of $1 \times 10^{5}$ cells $/ \mathrm{mL}$ and were treated with different concentrations of CGP57380 and everolimus alone or together for $24 \mathrm{~h}$ and $48 \mathrm{~h}$, respectively. On termination, the medium was replaced with fresh medium containing $0.5 \mathrm{mg} / \mathrm{mL}$ of $\mathrm{MTT}$. The cells were incubated for an additional $4 \mathrm{~h}$ at $37^{\circ} \mathrm{C}$. Following removal of the medium and MTT, DMSO $(200 \mu \mathrm{L})$ was added to each well, and the absorbance of the reaction was measured at $570 \mathrm{~nm}$ by spectrophotometry. The drug concentrations required to inhibit $50 \%$ of cell growth $\left(\mathrm{IC}_{50}\right)$ was calculated using nonlinear regression analysis.

\section{Detection of apoptosis}

Cells were cultured at a density of $1 \times 10^{5} / \mathrm{mL}$ in a 6-well plate and were treated with each drug for $24 \mathrm{~h}$. Cell pellets were collected and fixed with $70 \%$ ethanol on ice for $20 \mathrm{~min}$, followed by centrifugation. Apoptotic cells were quantified by propidium iodide (PI) and Annexin V-FITC double staining using a detection kit (BD Pharmingen, San Diego, CA, USA) according to the manufacturer's instructions. Samples were analyzed by flow cytometry (Accuri C6; BD, Franklin Lakes, NJ, USA).

\section{Western blot analysis}

The cells treated using various concentrations of drugs that were lysed at $4{ }^{\circ} \mathrm{C}$ in lysis buffer. The protein concentration was determined using the bicinchoninic acid (BCA) method. Equal amounts of total cell lysates were separated on sodium dodecylsulfate (SDS)-polyacrylamide gels containing 8\%-12\% acrylamide gradients and then were transferred to polyvinylidene difluoride membranes (Millipore, Bedford, MA, USA). The membranes were blocked for $2 \mathrm{~h}$ in Tris-buffered saline containing $0.1 \%$ Tween and $5 \%$ nonfat dry milk and then were incubated with primary antibodies overnight at $4{ }^{\circ} \mathrm{C}$. After incubation with horseradish peroxidase-conjugated secondary antibodies (1:5000; MultiSciences Biotech), the results were visualized using the $E C L$ detection kit (Biological Industries, Beit Ahemeq, Israel). All primary antibodies have been described previously.

\section{Nuclear and cytoplasmic extraction}

The cytoplasmic and nuclear proteins were extracted using NEPER Nuclear and Cytoplasmic Extraction Reagents (Thermo Fisher Scientific, Rockford, IL, USA) according to the manufacturer's instructions. Everolimus-treated cells were pelleted by centrifugation and rinsed with PBS and then were resuspended in ice-cold Cytoplasmic Extraction Reagent I, followed by vortexing the tube for $15 \mathrm{~s}$. After incubation on ice for $10 \mathrm{~min}$, Cytoplasmic Extraction Reagent II was added to the tube, which was then vortexed, incubated and centrifuged at maximum speed $(\sim 16,000 \times g)$ for $5 \mathrm{~min}$. The supernatant was transferred to a fresh tube and was referred to as the cytoplasmic extract. The insoluble fraction, containing nuclei, was suspended in ice-cold nuclear extraction reagent, placed on ice and vortexed continuously at the highest setting for $15 \mathrm{~s}$ every $10 \mathrm{~min}$, for a total of $40 \mathrm{~min}$. After centrifugation at maximum speed $(\sim 16000 \times g)$ for $10 \mathrm{~min}$, the supernatant was transferred to a new tube and was referred to as the nuclear fraction.

\section{Immunofluorescence assays}

Cells after drug treatment were fixed with freshly prepared $4 \%$ paraformaldehyde in PBS for 30 min at room temperature in the centrifuge tube. Next, the cells were washed with PBS 3 times, permeabilized with $0.3 \%$ Triton $\mathrm{X}-100$ in PBS (pH 7.2) for $10 \mathrm{~min}$ and blocked with PBS containing $10 \%$ goat serum for $30 \mathrm{~min}$ at room temperature. The cells were then stained with rabbit monoclonal antibody against phosphor-elF4E (Ser209) (1:200, Abcam) in PBS containing $1 \%$ BSA overnight at $4{ }^{\circ} \mathrm{C}$. A DyLight 488 goat anti-rabbit polyclonal antibody (Abcam) at $1 / 500$ dilution was used as the secondary antibody for $1 \mathrm{~h}$ at $37^{\circ} \mathrm{C}$. 

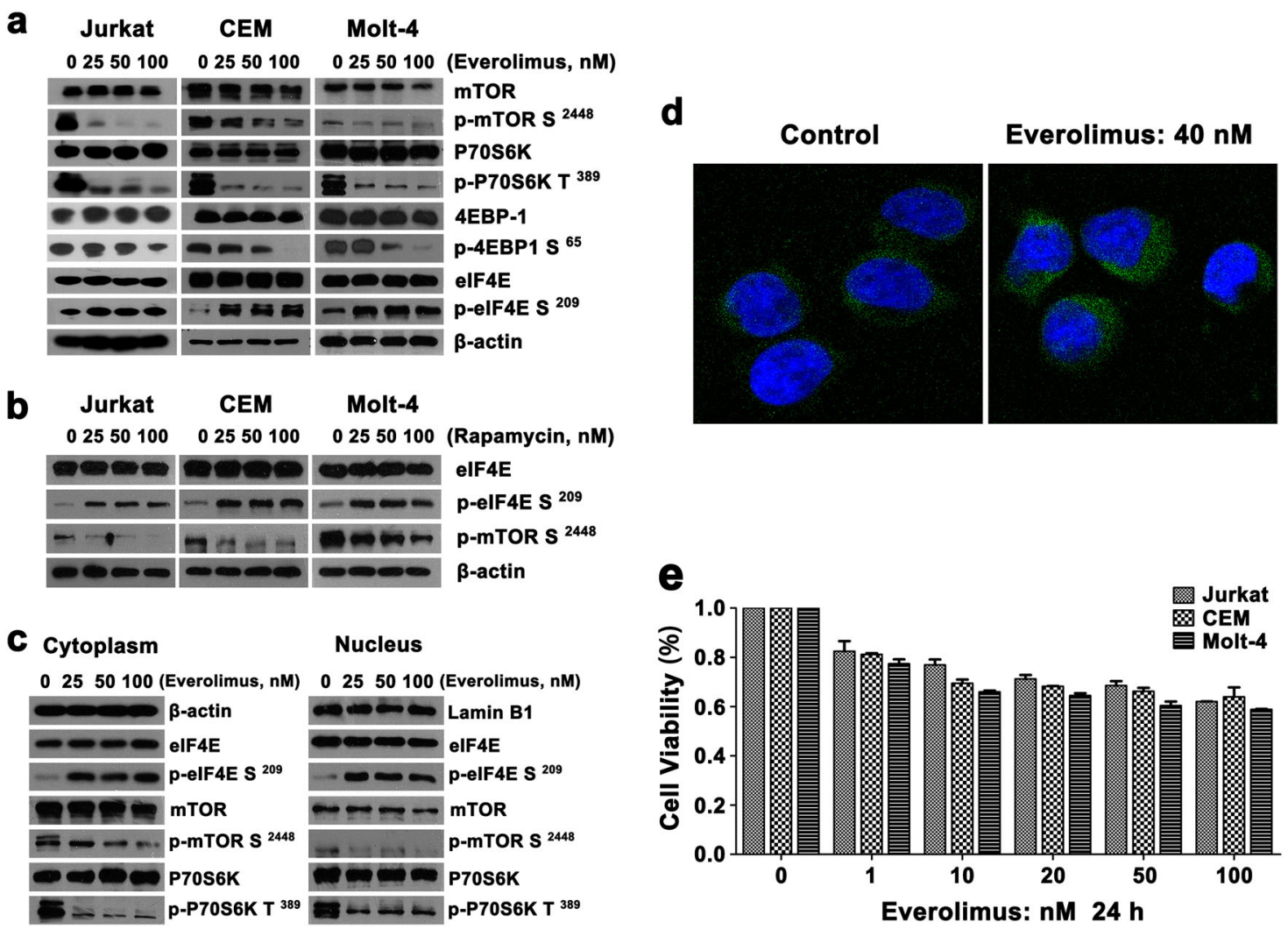

Fig. 1 Reactivation of elF4E and limited effects of everolimus on T-ALL cell lines. Jurkat, CEM and Molt-4 cells were treated with different concentrations of everolimus a or rapamycin b for $24 \mathrm{~h}$, and Western blotting analysis was performed for the expression of several proteins, including the phosphorylated and total levels of mTOR, 70S6K, 4EBP-1, and elF4E. c Cytoplasmic or nuclear protein extracts derived from Jurkat cells treated with everolimus at the indicated doses for $24 \mathrm{~h}$ were separated by SDS-polyacrylamide gel electrophoresis. Samples were immunoblotted with the indicated antibodies. d Jurkat cells were treated with everolimus ( $40 \mathrm{nM})$ for $24 \mathrm{~h}$ and then were stained with antiphosphor-elF4E, followed by incubation with secondary antibody conjugated with DyLight 488 (Green). The nuclei were stained with DAPI (Blue). Signals were detected by confocal microscopy. The merged panels show overlapping images of the two fluorescing signals. e The three T-ALL cell lines were incubated with various concentrations of everolimus (1-100 nM) for 24 $h$, and the MTT assay was used to assess cell viability. All the data are representative of 3 determinations with identical results

After washing 3 times with PBS ( $\mathrm{pH} 7.2)$, the nuclei were counterstained with $1 \mu \mathrm{M}$ of DAPI (4Z, 6Z-diamidino-2-phenylindole; Sigma-Aldrich) for $10 \mathrm{~min}$. The fluorescence was observed under a Nikon-Si Confocal Laser Scanning Microscope.

mTOR and MNK1 knockdown by short hairpin RNA

Three recombinant lentivirus vectors containing shRNA against mTOR or MNK1, and a negative control scrambled shRNA were purchased from Hanheng Biotech (Shanghai, China). Jurkat cells $\left(1 \times 10^{4}\right.$ cells/well) were seeded in a 6 -well plate and were transfected with lentivirus at a multiplicity of infection (MOI) of 100. After centrifugation at $1000 \times g$ for $1 \mathrm{~h}$, the cells were cultured at $37^{\circ} \mathrm{C}$. Next, the cells were selected in medium containing $2 \mu \mathrm{g} / \mathrm{mL}$ of puromycin (Gibco). The cellular viability and protein expression of mTOR- or MNK1-knockdown Jurkat cells were measured by the MTT assay and Western blotting. The sequence of shRNA targeting mTOR was $5^{\prime}$-CCCGGATCATTCACCCT ATTG-3', and the sequence of MNK1-shRNA was 5'-GGGATGA AACTGAACAACTCCTGTA-3'.

Statistical analysis

The data were analyzed by ANOVA and Student's $t$ test. $P<0.05$ was considered to indicate a statistically significant result. The synergy of everolimus with CGP57380 was analyzed using CalcuSyn software (Biosoft, Cambridge, UK). The data were expressed as $\log 10(\mathrm{Cl})$ vs the fraction affected. Using this method, $\log 10(\mathrm{Cl})<0$ indicates a synergistic effect.

\section{RESULTS}

Reactivation of elF4E and limited effects of everolimus against T-ALL cells

Everolimus, like rapamycin, is an mTORC1 inhibitor [12, 16]. To investigate whether the same inhibitory effects also exist in human T-ALL cells, Jurkat, CEM, and Molt-4 cells were treated with increasing concentrations of everolimus and rapamycin, respectively, for $24 \mathrm{~h}$. Cell lysates were assessed by immunoblotting for the activities of mTORC1 and its downstream molecules. Everolimus significantly inhibited $\mathrm{mTORC1}$, as indicated by the decrease in the phosphorylation of downstream effectors. The phosphorylation levels of Ser2448 on mTOR and Thr389 on p70S6K were inhibited in all the tested cell lines. However, everolimus induced increased elF4E phosphorylation (Fig. 1a). Similar results were seen when rapamycin was used in this experiment (Fig. 1b). elF4E in the cytoplasm is critical to initiate the translation of most mRNAs, while elF4E in the nucleus may enhance export to the cytoplasm of certain mRNAs $[12,16]$. By confocal microscopy and Western blot analyses, we evaluated the levels of phosphorylation of elF4E in Jurkat cells. Everolimus increased the level of phosphor-elF4E on Ser209 both in the cytosolic fraction and nuclear fraction (Fig. 1c, d). Finally, we examined cell survival upon treatment with everolimus by the MTT assay. Although everolimus treatment inhibited the growth of each cell line in a dose-dependent manner, its antileukemia activity leveled off at $100 \mathrm{nM}$ with a maximum inhibition of $<40 \%$ of cell viability (Fig. 1 e). 
a
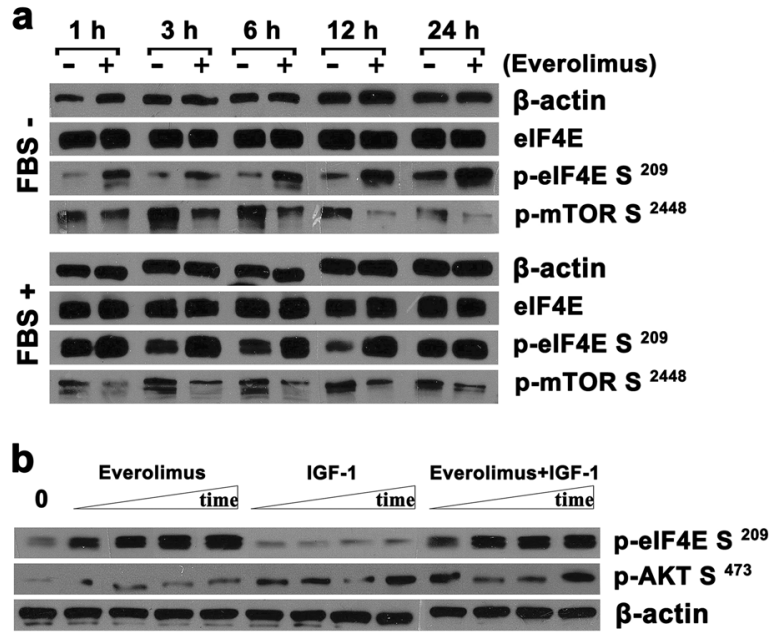

$\mathbf{c}$
$3 \mathrm{~h}$

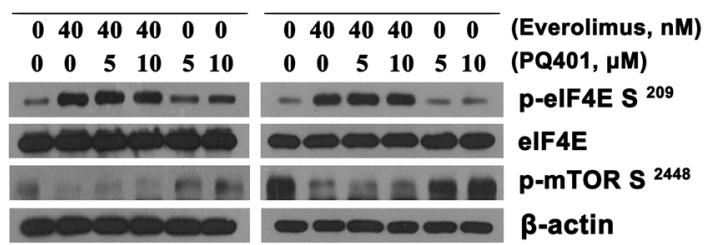

$\begin{array}{llllllllll}\text { d } & 0 & 0 & 10 & 20 & 40 & 10 & 20 & 40 & \text { (LY294002, } \mu M)\end{array}$

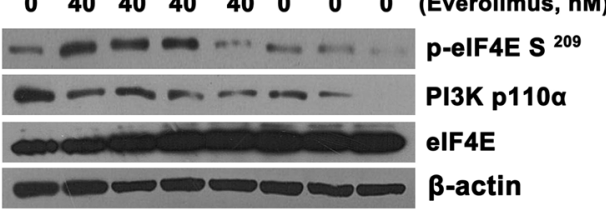

Fig. 2 Activation of elF4E induced by everolimus is independent of the growth factors but can be inhibited by a PI3K inhibitor. a Jurkat cells were serum starved for $24 \mathrm{~h}$ and then were treated with $40 \mathrm{nM}$ of everolimus in the absence or presence of FBS for the indicated times. $\mathbf{b}$ The cells were cultured in serum-deficient medium for $24 \mathrm{~h}$ and then were treated with $40 \mathrm{nM}$ of everolimus, $20 \mathrm{ng} / \mathrm{mL}$ of IGF-1, or their combination for $60,120,180$ and 360 min, respectively. c Jurkat cells were pretreated with $10 \mu M$ of PQ401 (IGF-1R inhibitor) for 60 min and then were co-treated with everolimus $(40 \mathrm{nM})$ for the indicated times. d Jurkat cells were incubated with different concentrations of everolimus and/or LY294002 for $24 \mathrm{~h}$. Whole-cell lysates were prepared and subjected to SDS-PAGE followed by immunoblotting with antibody that recognizes the corresponding antigen. The results are representative of three independent experiments
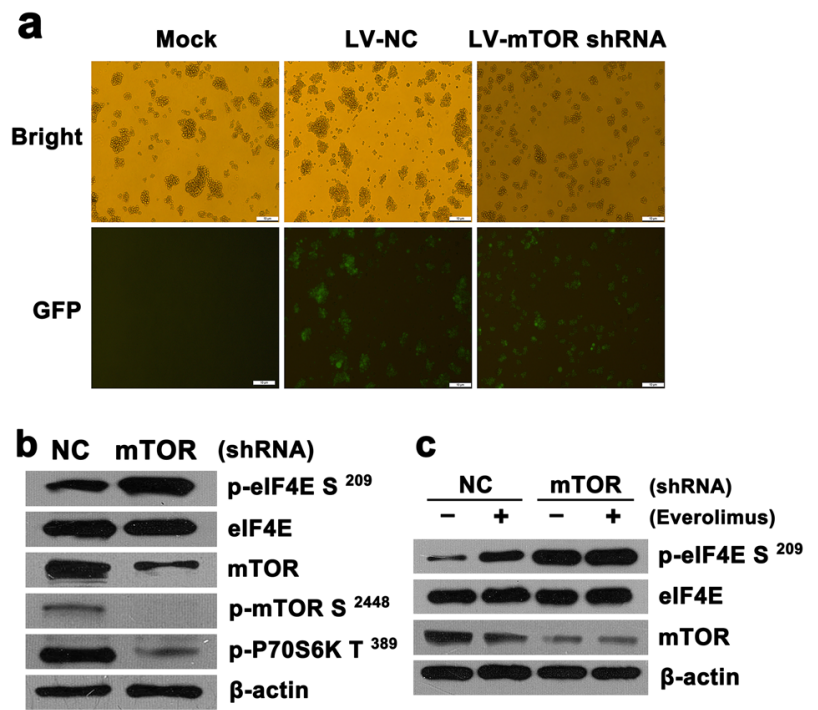

Fig. 3 Inhibition of mTOR contributes to the activation of elF4E. a Jurkat cells were infected with lentivirus carrying both GFP and mTOR shRNA (LV-mTOR shRNA) or nonsense shRNA (LV-NC) that served as a negative control. After puromycin selection, the expression of GFP was analyzed by fluorescence microscopy. b Jurkat cells stably transfected with shRNA anti-mTOR or control were analyzed for the expression of elF4E, phosphor-elF4E, mTOR, phosphor-mTOR, and phosphor-P70S6K by Western blotting. c Immunoblot analysis of mTOR and elF4E levels in Jurkat cells stably expressing a nonsilencing or MTOR shRNA and treated in the absence or presence of everolimus ( $40 \mathrm{nM}$ for $24 \mathrm{~h}$ )

Everolimus increases elF4E phosphorylation independently of growth factor stimulation and the IGF-1/IGF-1R axis

To determine whether everolimus-induced eIF4E phosphorylation was dependent on growth factor stimulation, we examined the effect of everolimus on the level of phosphor-elF4E in the absence of serum. For this purpose, Jurkat cells were cultured in serum-free medium for $24 \mathrm{~h}$, followed by everolimus treatment. As shown in Fig. 2a, everolimus treatment resulted in rapidly and persistently increased expression of phosphor-elF4E in the cells cultured with or without serum. It was reported that insulin-like growth factor-1 receptor (IGF-1R) supports cell growth/survival and contributes to aggressive biological behavior in a subset of human T-ALL [29, 30]. Thus, we investigated the potential molecular mechanism of the upregulation of phosphor-elF4E by examining the effect of insulinlike growth factor-1 (IGF-1) and IGF-1R inhibitor on everolimusinduced elF4E phosphorylation. Neither IGF-1 nor the IGF-1R inhibitor $\mathrm{PQ} 401$ altered the modulation of elF4E phosphorylation by everolimus (Fig. 2b, c). Finally, we also analyzed the effects of everolimus combined with LY294002, a PI3K inhibitor, on elF4E phosphorylation. As shown in Fig. 2d, in Jurkat cells, LY294002 was effective in downregulating PI3K p110a and dephosphorylating elF4E in a dose-dependent manner. A high concentration of LY294002 $(40 \mu \mathrm{M})$ was found to reverse everolimus-induced elF4E phosphorylation (Fig. 2d).

Inhibition of mTOR contributes to elF4E phosphorylation To determine a correlation between elF4E phosphorylation and the inhibition of mTOR, Jurkat cells were stably transfected with a lentivirus expressing a short hairpin RNA (shRNA) targeting mTOR or control, and the impact on elF4E phosphorylation was examined. GFP immunofluorescence demonstrated the transduction efficiency in Jurkat cells (Fig. 3a). Next, the gene knockdown efficiency was analyzed by Western blotting. Using this technique, we observed that mTOR expression was significantly reduced in Jurkat cells. We further observed that the silencing of mTOR resulted in markedly decreased phosphorylation of mTOR on Ser2448 and P70S6K on Thr389, a downstream target of mTORC1. Importantly, we also found that MTOR shRNA significantly increased the phosphorylation of elF4E (Fig. 3b). In addition, everolimus induced enhanced elF4E phosphorylation in mTORsilenced cells compared with the control (Fig. 3c).

The MNK1 inhibitor CGP57380 exhibits antileukemia activity The antiproliferative activity of CGP57380 was determined in T-ALL cell lines using the MTT assay. As shown in Fig. 4a, CGP57380 inhibited the proliferation of Jurkat and CEM cells in a dose- and time-dependent manner, with half-maximal inhibitory concentrations $\left(\mathrm{IC}_{50}\right)$ of 6.32 and $4.09 \mu \mathrm{M}$, respectively, at $48 \mathrm{~h}$. To characterize the cytotoxicity of CGP57380 against T-ALL cell lines, 

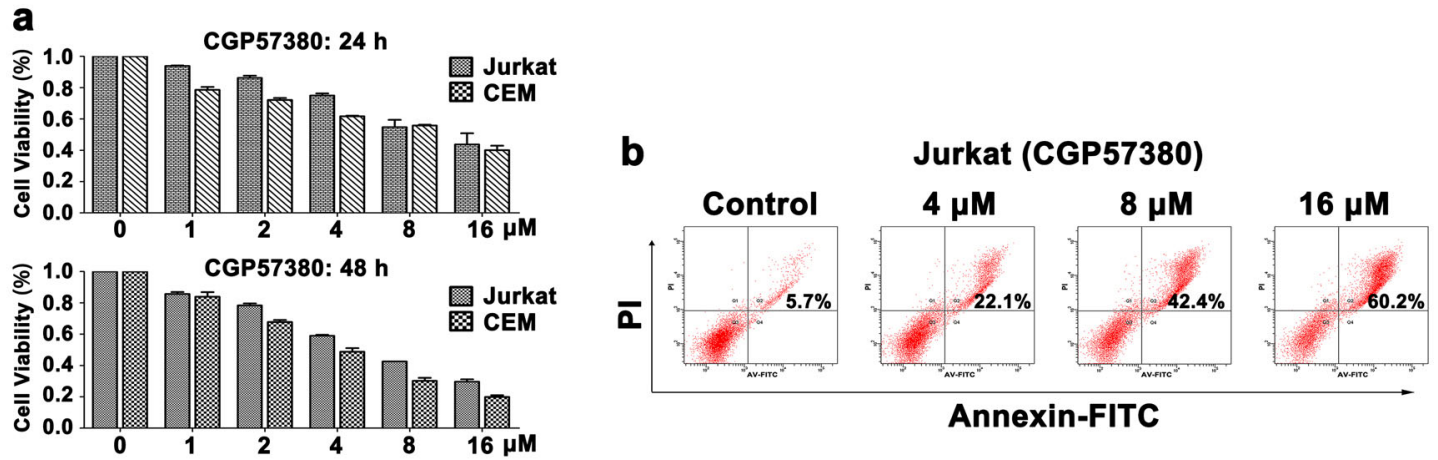

Annexin-FITC
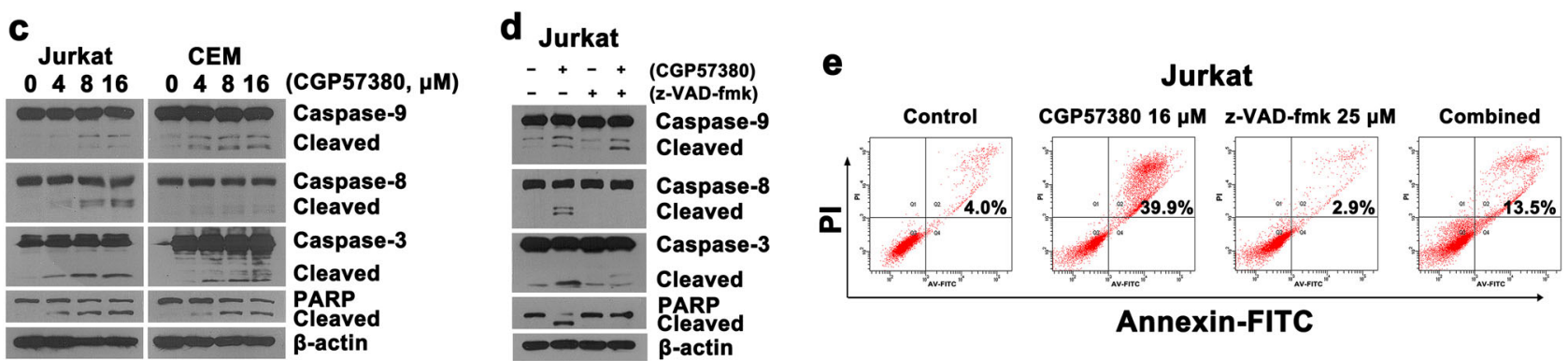

Annexin-FITC

Fig. 4 Effect of the MNK1 inhibitor CGP57380 on T-ALL cell lines. a Jurkat and CEM cells were cultured with increasing concentrations of CGP57380 for 24 and $48 \mathrm{~h}$, respectively. Cell viability was assessed by the MTT assay. b Jurkat cells were harvested after incubation with CGP57380 (4-16 $\mu \mathrm{M})$ for $24 \mathrm{~h}$, then cell apoptosis was assessed by the Annexin V/PI-staining-based flow cytometry assay. c The cleavage of caspase-3, $-8,-9$, and PARP was determined by Western blotting analysis after treatment with CGP57380 $(4,8$ and $16 \mu \mathrm{M})$ for $24 \mathrm{~h}$. d Jurkat cells were treated with $16 \mu \mathrm{M}$ CGP57380 for $24 \mathrm{~h}$, with or without $25 \mu \mathrm{M}$ z-VAD-fmk pretreatment. Activation of the caspase cascade was determined by Western blotting analysis. e Induction of apoptosis was assessed by flow cytometry after Annexin V/PI-staining

we next performed Annexin $\mathrm{V}$ and PI staining followed by flow cytometry analyses. Treatment with $16 \mu \mathrm{M}$ of CGP57380 induced apoptosis in more than $60 \%$ of Jurkat cells (Fig. 4b). We further used immunoblotting to assess the activation of caspases in Jurkat and CEM cell lines. As shown in Fig. 4c, CGP57380 triggered dose dependently the cleavage of caspase- $9,-8,-3$ and PARP. Moreover, activation of the caspase pathway induced by CGP57380 was almost completely blocked by pretreatment with the pan-caspase inhibitor z-VAD-fmk $(25 \mu \mathrm{M})$ in Jurkat cells (Fig. 4d), and flow cytometry analysis showed that the cytotoxicity against Jurkat cells was significantly reduced by z-VAD-fmk pretreatment: the percentage values of apoptotic cells were $4 \%, 39.9 \%$, and $13.5 \%$ in the control, $4 \mu \mathrm{M}$ CGP57380, and z-VAD-fmk followed by $4 \mu \mathrm{M}$ CGP57380 groups, respectively (Fig. 4e).

CGP57380 inhibits the phosphorylation of MNK1 and downstream elF4E in T-ALL cells

It was reported that CGP5.7380, a potent MNK1 and MNK2 inhibitor, substantially reduced elF4G in the elF4F complex, thereby inhibiting elF4E phosphorylation [31]. Thus, Jurkat and CEM cell lines treated with CGP57380 showed progressive dephosphorylation of MNK1 and elF4E (Fig. 5a). This finding was confirmed by confocal microscopy analysis (Fig. 5b). To further understand the consequences of specific MNK1 inhibition, we knocked down MNK1 using a lentiviral shRNA vector in Jurkat cells. We observed a significant decrease not only in the expression of MNK1 and phosphor-MNK1 but also in the expression of phosphor-elF4E in Jurkat cells transfected with MNK1 shRNA (Fig. 5c). Because the phosphorylation of elF4E, a key component of the mRNA cap-binding complex, by MNK1 may enhance the translation of some mRNAs to produce cancerassociated proteins, including anti-apoptotic proteins, such as Mcl-1 and Bcl-2, and oncoproteins, such as c-Myc and cyclin D1 $[21,31]$, we next examined the effects of CGP57380 on the expression of these anti-apoptotic proteins and oncoproteins (Fig. 5d). A significant reduction in the levels of Mcl-1, c-Myc and surviving, but slight downregulation of $\mathrm{Bcl}-2$ and cyclindependent kinase-2 (CDK-2) expression, was observed.

CGP57380 overcomes everolimus-mediated elF4E phosphorylation and sensitizes T-ALL cells to everolimus In the last group of experiments, we tested whether the therapeutic strategy combining CGP57380 with everolimus could be effective in treating T-ALL. Jurkat and CEM cells were treated with CGP57380 $(2-16 \mu \mathrm{M})$ combined with everolimus (5-40 nM). As shown in Fig. $6 a, b$, the combination with CGP57380 and everolimus was more efficacious than either single drug in inhibiting the cellular proliferation of T-ALL cell lines. The $\mathrm{Cl}$ at the median effective dose $\left(E_{50}\right)$ was 0.21 for Jurkat cells (Fig. 6b), further indicating that everolimus and CGP57380 cotreatment is highly synergistic. Apoptosis was also quantified using the PI staining assay and Annexin $\mathrm{V}$ binding. The combination treatment elicited $33.5 \%$ of Jurkat cells to undergo apoptosis, whereas treatment with everolimus or CGP57380 alone resulted in $7.4 \%$ and $20.8 \%$ of apoptotic cells, respectively (Fig. $6 \mathrm{c}$ ). Similar results were obtained with CEM cells (Fig. 6c). Next, the expression of caspase pathway molecules was also examined after everolimus and CGP57380 treatment. As shown in Fig. 6d, combined but not individual treatment resulted in a pronounced increase in the cleavage of caspase- 3 , caspase- 8 and caspase- 9 and degradation of PARP in Jurkat and CEM cells. To investigate whether CGP57380 indeed blocked everolimus-induced eIF4E phosphorylation, we further treated Jurkat cells with everolimus in the absence and presence of CGP57380 and analyzed elF4E phosphorylation by Western blotting analysis. As shown in Fig. 6e, the presence of CGP57380 effectively inhibited everolimus-induced elF4E phosphorylation. Finally, to demonstrate that the synergistic killing effect was preferentially mediated by MNK1 inhibition, we then compared levels of elF4E phosphorylation between Jurkat cells and Jurkat cells with MNK1 shRNA after treatment with everolimus. The results revealed that the silencing of MNK1 weakened everolimus-induced elF4E phosphorylation (Fig. 6f). 

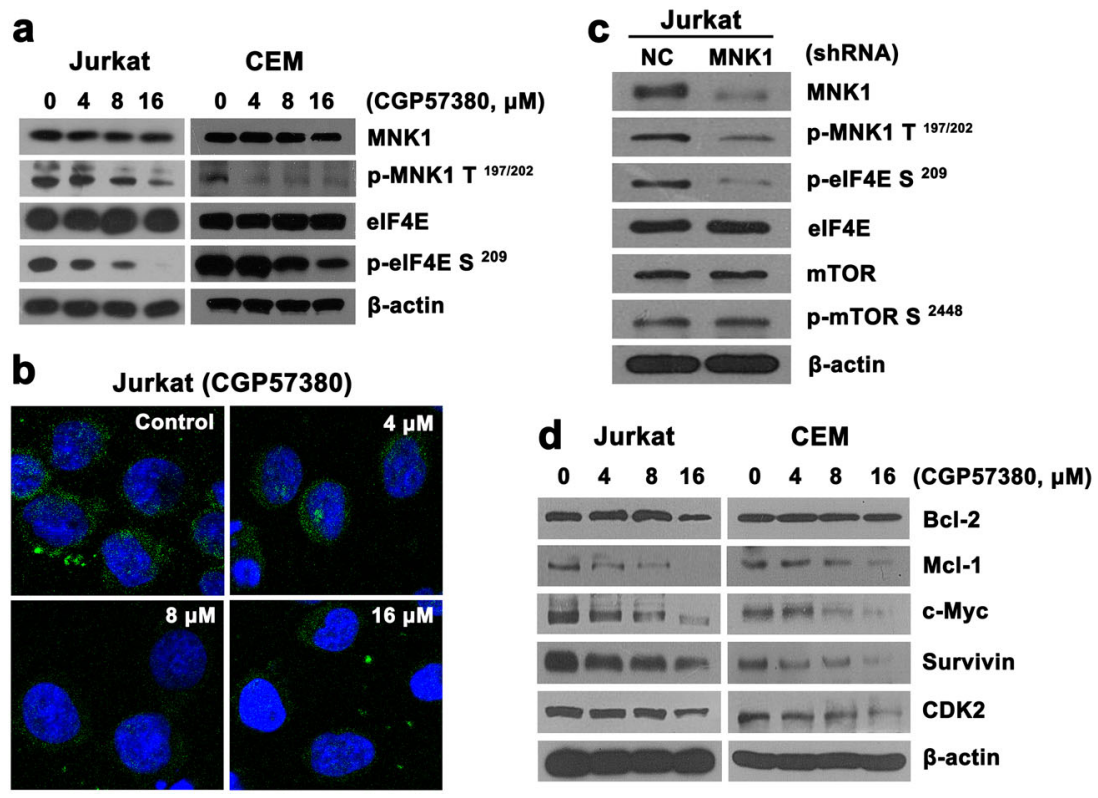

Fig. 5 CGP57380 blocks the phosphorylation of MNK1 and downstream elF4E in T-ALL cells. a Western blotting analysis of MNK1 and elF4E expression in Jurkat and CEM cells after treatment with different concentrations (4-16 $\mu \mathrm{M})$ of CGP57380 for $24 \mathrm{~h}$. b Confocal images of Jurkat cells stained with anti-phosphor-elF4E antibody, followed by incubation with DyLight 488 goat anti-rabbit polyclonal antibody. Fluorescence was visualized by confocal microscopy. c Western blotting analysis of MNK1, elF4E, and mTOR in Jurkat cells stably transfected with shRNA anti-MNK1 (LV-MNK1 shRNA) or nonsilencing control (LV-NC). d After treatment with CGP57380 at various concentrations for $24 \mathrm{~h}$, the expression of several downstream targets, such as Bcl-2, Mcl-1, c-Myc, survivin and CDK2, in Jurkat and CEM cells were determined by Western blotting analysis

\section{DISCUSSION}

The regulation of the PI3K/Akt/mTOR pathway is complex, largely due to the existence of multiple feedback loops and direct activation mechanisms that place mTOR both upstream and downstream of several oncogenic and antiapoptotic pathways $[6,7]$. For example, the mTORC1 inhibitor everolimus could hyperactivate Akt, which hampers its anti-cancer action and results in drug resistance [32-34]. On the other hand, it was also reported that the inhibition of mTOR signaling results in elF4E phosphorylation in human cancer cells, including lung cancer and breast cancer cells $[35,36]$. However, the issue of increased elF4E phosphorylation in response to MTORC1 inhibition has not been investigated in T-ALL. Previous studies have shown that most breast cancer cell lines are sensitive to everolimus with $\mathrm{IC}_{50}$ values of less than $20 \mathrm{nM}[37,38]$. In the present study, we found that, although everolimus inhibits the growth of T-ALL cell lines in a dose-dependent manner, its cytotoxicity leveled off at $100 \mathrm{nM}$ with a maximum inhibition below $40 \%$ of cell viability, indicating that T-ALL cells are relatively resistant to everolimus. Additionally, Jurkat cells exposed to everolimus exhibited increased elF4E phosphorylation at Ser209. In the shRNA experiment, silencing of mTOR also induced elF4E phosphorylation, clearly indicating a correlation between elF4E phosphorylation and the inhibition of mTOR in T-ALL cells. Taken together, everolimus-induced elF4E phosphorylation may contribute to weaken the anticancer efficacy of the mTORC1 inhibitor.

Previous studies have shown that rapamycin induced-elF4E phosphorylation is not observed in lung cancer and breast cancer cells where both MNK1 and MNK2 were knocked out, suggesting that increased elF4E phosphorylation by mTOR inhibitor is mediated through an MNK-dependent pathway $[35,36]$. In human medulloblastoma and prostate cancer cells, MNK2, but not MNK1, contributes to the effect of mTORC1 inhibitors on elF4E phosphorylation $[39,40]$. Our study shows that the activation of elF4E induced by everolimus is MNK1 mediated; however, silencing of the MNK1 gene only partially alters the modulation of elF4E phosphorylation by everolimus. "Thus, whether MNK2 is linked to activation of elF4E in T-ALL cells needs to be investigated in the future". We also found that the presence of the PI3K inhibitor, LY294002, remarkably abrogated elF4E phosphorylation induced by everolimus. This result is consistent with studies in different model systems [35]. Given these observations, it could be speculated that triple combinations of a PI3K inhibitor, an mTORC1 inhibitor, and an MNK1 inhibitor in the treatment of T-ALL might be a new and promising therapeutic approach.

It is well known that the phosphorylation of elF4E by MNK on Ser209 is critical for the oncogenic activity of elF4E $[18,28]$. Inhibition of MNK could suppress lymphogenesis driven by the loss of PTEN [41]. Thus, inhibiting MNKs might be the new therapeutic approach to target hyperactivated T-ALL cells treated with everolimus. In our present study, we investigated the effects of the MNK1 inhibitor CGP57380 on T-ALL cell lines. We found that CGP57380 suppressed the levels of phosphor-MNK1 and elF4E phosphorylation at Ser209 and inhibited cellular proliferation and induced apoptosis that was dependent on activation of the caspase pathway in T-ALL cells. NOTCH1 mutations have been found in $>50 \%$ of patients with T-ALL, indicating the NOTCH-1 gene is a major oncogenic driver and therapeutic target in T-ALL [42]. A more recent study demonstrated that the oncogenic activity of NOTCH-1 in T-ALL is strictly dependent on Myc upregulation [43]. In this study, it was observed that CGP57380 downregulates $\mathrm{Mcl}-1$, survivin, and c-Myc. These data collectively demonstrate multiple mechanisms seem to be implicated in the antileukemic activity of CGP57380, possibly contributing to the combination effect of CGP57380 and everolimus.

In summary, we demonstrated that the MNK1 inhibitor CGP57380 can overcome the limitation of activation of elF4E induced by everolimus. CGP57380 also significantly inhibits the expression of MNK1 and phophor-MNK1, thereby downregulating its downstream molecules, including Mcl-1, c-Myc, survivin, and CDK2. Furthermore, CGP57380 sensitizes T-ALL cells to apoptosis induced by everolimus. Thus, our results suggest that the 

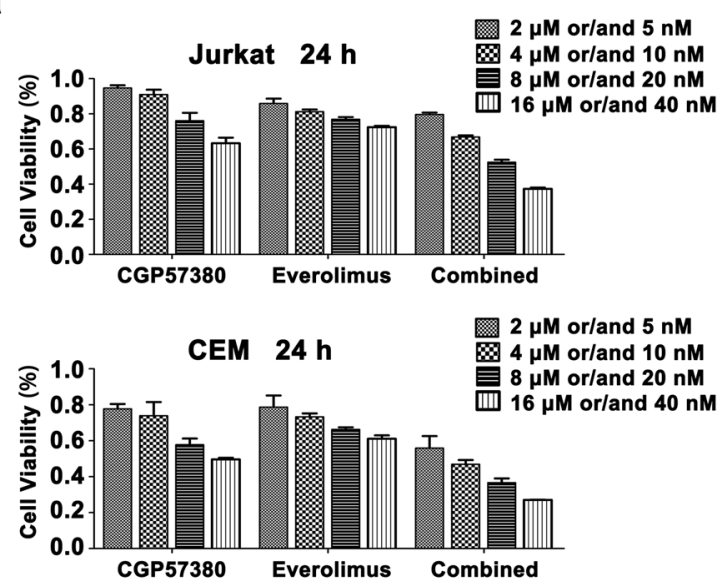

C

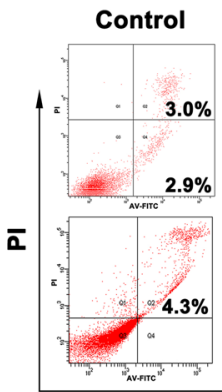

CGP57380

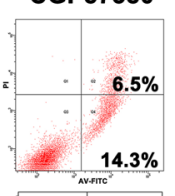

\section{Everolimus}

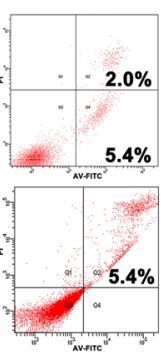

Combined

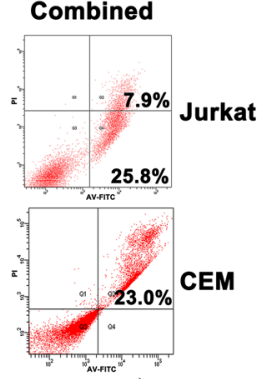

Annexin-FITC

e

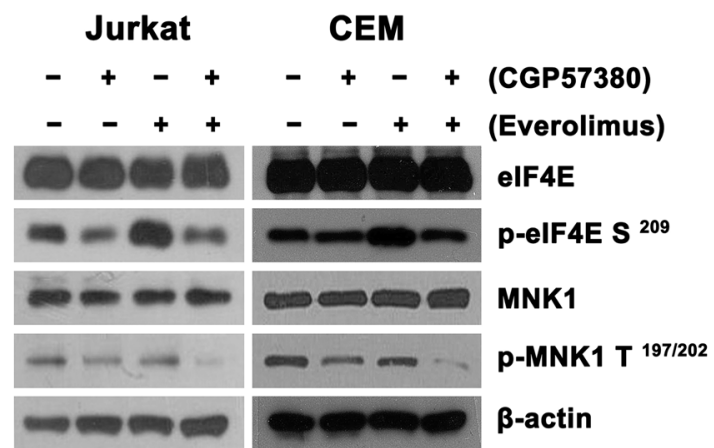

b
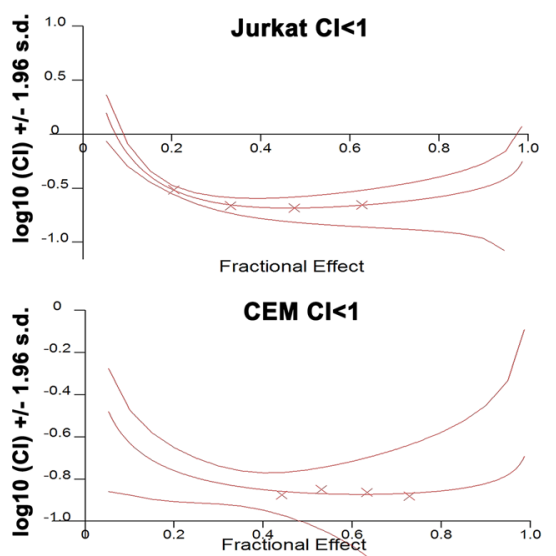

d Jurkat CEM

- + + - + - + (CGP57380)

-+++-++ (Everolimus)

$\longrightarrow$ B-actin

Deberase-9

Cleaved

$\square \square \square=0$ Caspase-8

$+\infty=\square=$ Cleaved

$\longrightarrow$ Caspase-3

Cleaved

PARP

Cleaved

\section{f}

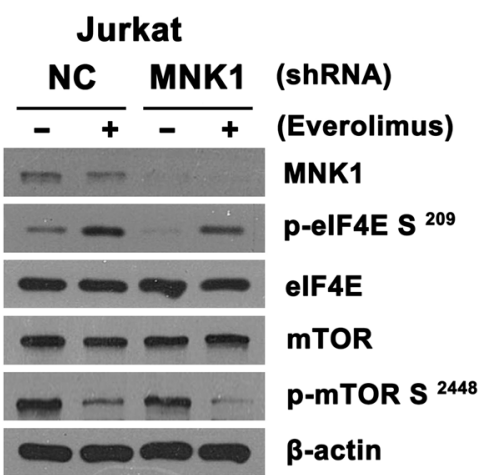

Fig. 6 The combination of CGP57380 with everolimus overcomes everolimus-mediated elF4E phosphorylation and shows enhanced cytotoxicity against T-ALL cells. a, b Jurkat and CEM cells were treated with a series of doses of CGP57380 and/or everolimus for $24 \mathrm{~h}$, and cell viability was measured by the MTT assay. The data are presented as the means \pm SD from three independent experiments. The combination index was calculated by Calcusyn software and was expressed as log $10(\mathrm{Cl})$ vs fraction affected. Where calculable, $95 \%$ confidence intervals are shown. c Jurkat and CEM cells were incubated with everolimus ( $20 \mathrm{nM})$, CGP57380 $(4 \mu \mathrm{M})$, or in combination for $24 \mathrm{~h}$, followed by Annexin V/PI staining and flow cytometry to detect apoptosis. d Jurkat and CEM cells were treated with everolimus (20 nM) alone or in combination with CGP57380 $(4 \mu \mathrm{M})$ for $24 \mathrm{~h}$. Cleavage of PARP, caspase-3, caspase-8, and caspase- 9 was analyzed by Western blotting analysis. e Jurkat and CEM cells were treated with CGP57380 $(4 \mu \mathrm{M})$, everolimus $(20 \mathrm{nM})$ or in combination for $24 \mathrm{~h}$. The expression and phosphorylation of MNK1 and elF4E were determined by Western blotting analysis. $\mathbf{f}$ Immunoblot analysis of mTOR, elF4E, MNK1 expression and the phosphorylation levels in Jurkat cells stably expressing a nonsilencing or MNK1 shRNA. The cells were treated in the absence or presence of everolimus ( $40 \mathrm{nM}$ for $24 \mathrm{~h}$ )

combination of targeting both mTOR and MNK1/elF4E is an attractive strategy for T-ALL.

\section{ACKNOWLEDGEMENTS}

The research was supported by funds from the Science Technology Department of Zhejiang Province (No. 2016 C33137 and No. 2018C03016-1), National Natural Science Foundation of China (No. 81670178), The National Key Research and Development
Program of China (No. 2016YFC090150X), and Research Project for Practice Development of National TCM Clinical Research Bases (No. JDZX2015113).

\section{AUTHOR CONTRIBUTION}

W-bQ and X-jY designed the study; X-bH and C-mY conducted the study; X-bH, Q-mH and WL contributed new analytical tools and reagents; $\mathrm{X}$-bH analyzed the data; $\mathrm{X}-\mathrm{bH}$ and $W$-bQ wrote the paper. All authors participated in the drafting of the manuscript and approved its final version. 


\section{ADDITIONAL INFORMATION}

Conflict of interest: The authors declare that they have no competing interest.

\section{REFERENCES}

1. Jabbour E, O'Brien S, Konopleva M, Kantarjian H. New insights into the pathophysiology and therapy of adult acute lymphoblastic leukemia. Cancer. 2015;121:2517-28.

2. Ferrando AA, Neuberg DS, Staunton J, Loh ML, Huard C, Raimondi SC, et al. Gene expression signatures define novel oncogenic pathways in $\mathrm{T}$ cell acute lymphoblastic leukemia. Cancer Cell. 2002;1:75-87.

3. Pui $\mathrm{CH}$, Evans WE. Treatment of acute lymphoblastic leukemia. $\mathrm{N}$ Engl J Med. 2006;354:166-78.

4. Möricke A, Reiter A, Zimmermann M, Gadner H, Stanulla M, Dördelmann M, et al. Risk-adjusted therapy of acute lymphoblastic leukemia can decrease treatment burden and improve survival: treatment results of 2169 unselected pediatric and adolescent patients enrolled in the trial ALL-BFM 95. Blood. 2008;111:4477-89.

5. DeAngelo DJ, Stevenson KE, Dahlberg SE, Silverman LB, Couban S, Supko JG, et al. Long-term outcome of a pediatric-inspired regimen used for adults aged 18-50 years with newly diagnosed acute lymphoblastic leukemia. Leukemia. 2015;29:526-34

6. Evangelisti C, Evangelisti C, Chiarini F, Lonetti A, Buontempo F, Bressanin D, et al. Therapeutic potential of targeting mTOR in T-cell acute lymphoblastic leukemia (review). Int J Oncol. 2014;45:909-18.

7. Zhao WL. Targeted therapy in T-cell malignancies: dysregulation of the cellular signaling pathways. Leukemia. 2010;24:13-21.

8. Martelli AM, Chiarini F, Evangelisti C, Cappellini A, Buontempo F, Bressanin D, et al. Two hits are better than one: targeting both phosphatidylinositol 3-kinase and mammalian target of rapamycin as a therapeutic strategy for acute leukemia treatment. Oncotarget. 2012;3:371-94.

9. Laplante M, Sabatini DM. mTOR signaling at a glance. J Cell Sci. 2009;122: 3589-94.

10. Hoshii T, Kasada A, Hatakeyama T, Ohtani M, Tadokoro $Y$, Naka K, et al. Loss of mTOR complex 1 induces developmental blockage in early T-lymphopoiesis and eradicates T-cell acute lymphoblastic leukemia cells. Proc Natl Acad Sci U S A. 2014;111:3805-10.

11. Ikezoe T, Nishioka C, Bandobashi K, Yang $Y$, Kuwayama $Y$, Adachi $Y$, et al. Longitudinal inhibition of PI3K/Akt/mTOR signaling by LY294002 and rapamycin induces growth arrest of adult T-cell leukemia cells. Leuk Res. 2007;31:673-82.

12. Zhao $Y M$, Zhou $Q, X u ~ Y$, Lai $X Y$, Huang $H$. Antiproliferative effect of rapamycin on human T-cell leukemia cell line Jurkat by cell cycle arrest and telomerase inhibition. Acta Pharmacol Sin. 2008;29:481-8.

13. Batista A, Barata JT, Raderschall E, Sallan SE, Carlesso N, Nadler LM, et al Targeting of active mTOR inhibits primary leukemia $T$ cells and synergizes with cytotoxic drugs and signaling inhibitors. Exp Hematol. 2011;39:457-72.

14. Wu KN, Zhao YM, He Y, Wang BS, Du KL, Fu S, et al. Rapamycin interacts synergistically with idarubicin to induce T-leukemia cell apoptosis in vitro and in a mesenchymal stem cell simulated drug-resistant microenvironment via Akt/mammalian target of rapamycin and extracellular signal-related kinase signaling pathways. Leuk Lymphoma. 2014;55:668-76.

15. Meng LH, Zheng XF. Toward rapamycin analog (rapalog)-based precision cancer therapy. Acta Pharmacol Sin. 2015;36:1163-9.

16. Witzig TE, Reeder C, Han JJ, LaPlant B, Stenson M, Tun HW, et al. The mTORC inhibitor everolimus has antitumor activity in vitro and produces tumor responses in patients with relapsed T-cell lymphoma. Blood. 2015;126:328-35.

17. Daver N, Boumber Y, Kantarjian H, Ravandi F, Cortes J, Rytting ME, et al. A phase $I / I I$ study of the mTOR inhibitor everolimus in combination with HyperCVAD chemotherapy in patients with relapsed/refractory acute lymphoblastic leukemia. Clin Cancer Res. 2015;21:2704-14.

18. Buxade M, Parra-Palau JL, Proud CG. The Mnks: MAP kinase-interacting kinases (MAP kinase signal-integrating kinases). Front Biosci. 2008;13:5359-73.

19. Wendel HG, De Stanchina E, Fridman JS, Malina A, Ray S, Kogan S, et al. Survival signalling by $A k t$ and elF4E in oncogenesis and cancer therapy. Nature. 2004;428:332-7.

20. Ueda T, Watanabe-Fukunaga R, Fukuyama H, Nagata S, Fukunaga R. Mnk2 and Mnk1 are essential for constitutive and inducible phosphorylation of eukaryotic initiation factor $4 \mathrm{E}$ but not for cell growth or development. Mol Cell Biol. 2004;24:6539-49.
21. Li P, Diab S, Yu M, Adams J, Islam S, Basnet SK, et al. Inhibition of Mnk enhances apoptotic activity of cytarabine in acute myeloid leukemia cells. Oncotarget. 2016;7:56811-25

22. Kosciuczuk EM, Saleiro D, Kroczynska B, Beauchamp EM, Eckerdt F, Blyth GT, et al. Merestinib blocks Mnk kinase activity in acute myeloid leukemia progenitors and exhibits antileukemic effects in vitro and in vivo. Blood. 2016;128:410-4.

23. Wu H, Hu C, Wang A, Weisberg EL, Chen Y, Yun $\mathrm{CH}$, et al. Discovery of a BTK/MNK dual inhibitor for lymphoma and leukemia. Leukemia. 2016;30:173-81.

24. O'Reilly $K E$, Rojo F, She QB, Solit D, Mills GB, Smith D, et al. mTOR inhibition induces upstream receptor tyrosine kinase signaling and activates Akt. Cancer Res. 2006;66:1500-8.

25. Carracedo A, Ma L, Teruya-Feldstein J, Rojo F, Salmena L, Alimonti A, et al Inhibition of mTORC1 leads to MAPK pathway activation through a PI3Kdependent feedback loop in human cancer. J Clin Invest. 2008;118:3065-74.

26. Wang X, Hawk N, Yue P, Kauh J, Ramalingam SS, Fu H, et al. Overcoming mTOR inhibition-induced paradoxical activation of survival signaling pathways enhances mTOR inhibitors' anticancer efficacy. Cancer Biol Ther. 2008;7:1952-8.

27. Brunen D, García-Barchino MJ, Malani D, Jagalur Basheer N, Lieftink C, Beijersbergen RL, et al. Intrinsic resistance to PIM kinase inhibition in AML through p38a-mediated feedback activation of mTOR signaling. Oncotarget. 2016;7:37407-19.

28. Diab S, Kumarasiri M, Yu M, Teo T, Proud C, Milne R, et al. MAP kinase-interacting kinases-emerging targets against cancer. Chem Biol. 2014;21:441-52.

29. Gusscott S, Jenkins CE, Lam SH, Giambra V, Pollak M, Weng AP. IGF1R derived $\mathrm{PI3K} / \mathrm{AKT}$ signaling maintains growth in a subset of human T-cell acute lymphoblastic leukemias. PLoS One. 2016;11:e0161158.

30. Medyouf H, Gusscott S, Wang H, Tseng JC, Wai C, Nemirovsky O, et al. High-level IGF1R expression is required for leukemia-initiating cell activity in T-ALL and is supported by Notch signaling. J Exp Med. 2011;208:1809-22.

31. Hou J, Lam F, Proud C, Wang S. Targeting Mnks for cancer therapy. Oncotarget. 2012:3:118-31.

32. Seront E, Pinto A, Bouzin C, Bertrand L, Machiels JP, Feron O. PTEN deficiency is associated with reduced sensitivity to MTOR inhibitor in human bladder cancer through the unhampered feedback loop driving PI3K/Akt activation. Br J Cancer. 2013;109:1586-92.

33. Bertacchini J, Guida M, Accordi B, Mediani L, Martelli AM, Barozzi P, et al. Feedbacks and adaptive capabilities of the PI3K/Akt/mTOR axis in acute myeloid leukemia revealed by pathway selective inhibition and phosphoproteome analysis. Leukemia. 2014;28:2197-205.

34. Badura S, Tesanovic T, Pfeifer H, Wystub S, Nijmeijer BA, Liebermann M, et al Differential effects of selective inhibitors targeting the PI3K/AKT/mTOR pathway in acute lymphoblastic leukemia. PLoS One. 2013:8:e80070.

35. Wang $X$, Yue $P$, Chan $C B, Y e ~ K$, Ueda $T$, Watanabe-Fukunaga $R$, et al. Inhibition of mammalian target of rapamycin induces phosphatidylinositol 3-kinasedependent and Mnk-mediated eukaryotic translation initiation factor 4E phosphorylation. Mol Cell Biol. 2007;27:7405-13.

36. Wen Q, Wang WY, Luo JD, Chu SZ, Chen LJ, Xu LN, et al. CGP57380 enhances efficacy of RAD001 in non-small cell lung cancer through abrogating mTOR inhibition-induced phosphorylation of elF4E and activating mitochondrial apoptotic pathway. Oncotarget. 2016;7:27787-801.

37. Yunokawa $M$, Koizumi $F$, Kitamura $Y$, Katanasaka $Y$, Okamoto $N$, Kodaira $M$, et al Efficacy of everolimus, a novel mTOR inhibitor, against basal-like triple-negative breast cancer cells. Cancer Sci. 2012;103:1665-71.

38. Leung EY, Askarian-Amiri M, Finlay GJ, Rewcastle GW, Baguley BC. Potentiation of growth inhibitory responses of the mTOR inhibitor everolimus by dual mTORC1/2 inhibitors in cultured breast cancer cell lines. PLoS One. 2015;10:e0131400.

39. Stead RL, Proud CG. Rapamycin enhances elF4E phosphorylation by activating MAP kinase-interacting kinase 2a (Mnk2a). FEBS Lett. 2013;587:2623-8.

40. Eckerdt F, Beauchamp E, Bell J, lqbal A, Su B, Fukunaga R, et al. Regulatory effects of a Mnk2-elF4E feedback loop during mTORC1 targeting of human medulloblastoma cells. Oncotarget. 2014;5:8442-51.

41. Ueda T, Sasaki M, Elia AJ, Chio II, Hamada $K$, Fukunaga $R$, et al. Combined deficiency for MAP kinase-interacting kinase 1 and 2 (Mnk1 and Mnk2) delays tumor development. Proc Natl Acad Sci U S A. 2010;107:13984-90.

42. Weng AP, Ferrando AA, Lee W, Morris JP, Silverman LB, Sanchez-Irizarry C, et al. Activating mutations of NOTCH1 in human T cell acute lymphoblastic leukemia. Science. 2004;306:269-71.

43. Sanchez-Martin M, Ferrando A. The NOTCH1-MYC highway toward T-cell acute lymphoblastic leukemia. Blood. 2017;129:1124-33. 